\title{
Phage Therapy: Going Temperate?
}

\author{
Rodrigo Monteiro, ${ }^{1}$ Diana Priscila Pires, ${ }^{1}$ Ana Rita Costa, ${ }^{1,2, \star}$ and Joana Azeredo ${ }^{1, \star}$
}

Strictly lytic phages have been consensually preferred for phage therapy purposes. In contrast, temperate phages have been avoided due to an inherent capacity to mediate transfer of genes between bacteria by specialized transduction - an event that may increase bacterial virulence, for example, by promoting antibiotic resistance. Now, advances in sequencing technologies and synthetic biology are providing new opportunities to explore the use of temperate phages for therapy against bacterial infections. By doing so we can considerably expand our armamentarium against the escalating threat of antibiotic-resistant bacteria.

\section{The Awakening of Phage Therapy}

The last decades have witnessed the steady rise of antibiotic resistance as both a clinical and a public health problem [1]. In fact, antibiotic-resistant bacteria are predicted to kill 10 million people each year by $2050^{\prime}$. Importantly, there is also a growing fear that the unfeasibility of using antibiotics to treat bacterial infections will threaten routine medical procedures such as childbirth, blood transfusion, or minor surgeries like tonsillectomy, due to a high risk of postprocedure infections. It has thus become clear that new and more effective strategies are vital to prevent such a frightful future.

About 100 years ago, bacterial viruses called bacteriophages (phages) (see Glossary) were discovered by Felix d'Herelle and were immediately proposed for treating and preventing infectious diseases in both humans and animals [2]. At the time, d'Herelle envisioned a future in which phage therapy would be a major weapon in the fight against bacterial infections. But the discovery of the first antibiotic [3] rapidly caused the fall of phage therapy into oblivion. Now, with the threat of (multi-)antibiotic-resistant bacteria, d'Herelle's vision may actually become a reality.

Phages offer a few important advantages over antibiotics: a narrow spectrum of activity that protects the normal microbiota of the host, the capacity to multiply at the site of infection, being apparently harmless to humans, and lower discovery and production costs [4,5]. But, in order to use phages for therapeutic purposes, we need to consider that phages and bacteria have coevolved for millions of years, and that their interaction cannot lead to the extinction of one population. This implies that phages will never completely eliminate a target bacterial population on their own since a small subset of the bacterial population will evolve towards a phage-resistant phenotype. The immune system may here play a fundamental role in the success of phage therapy: neutrophil-phage synergy was shown to be essential for the efficient elimination of both phage-sensitive and emergent phage-resistant variants [6]. The combined use of phages with antibiotics or other antimicrobial strategies is also a viable option to overcome the ecological limitation of phage therapy. Moreover, phages display some other limitations that require the design of strategies to circumvent or eliminate these less favourable features. Synthetic biology has allowed for major advances in this area, having been successfully employed for modulating phage host range [7], reducing phage toxicity and immunogenicity $[8,9]$, enhancing phage

\section{Highlights}

Temperate phages are highly abundant in bacterial genomes and are more readily available than lytic phages. While temperate phages are not seen as suitable for therapeutic purposes, their natural or engineered lytic variants hold promise.

Advances in synthetic biology offer invaluable opportunities for the exploration of temperate phages in therapy, with the creation of lytic and tailored variants.

Innovative approaches emerged exploring the integration of temperate phages in bacterial genomes for therapeutic purposes, as the dissemination of antibiotic susceptibility.

Encouraging results have been reported from the use of temperate phages and their lytic variants in therapy, for example, reduced phageresistant variants and endotoxin/toxin release; but negative outcomes have also been described - for example, increased sporulation. A cautious approach to temperate phage therapy is still required.

${ }^{1} \mathrm{CEB}$ - Centre of Biological Engineering, University of Minho, Braga, Portugal

${ }^{2}$ Present address: Department of Bionanoscience, Kavli Institute of Nanoscience, Delft University of Technology, Delft, The Netherlands

${ }^{*}$ Correspondence:

a.r.martinscosta@tudelft.nl (A.R. Costa) and

jazeredo@deb.uminho.pt (J. Azeredo). 
survival after administration [10], improving phage activity against biofilms [11], and enhancing bacterial killing when combined with antibiotics [12].

Recommendations for phage therapy advise the choice of strictly lytic phages (Box 1), mostly to avoid potential transfer of bacterial antibiotic resistance or virulence genes by temperate phages (Box 1) via transduction [13,14]. However, this guideline was set before the development of efficient approaches for engineering phage genomes. Now, synthetic biology can be used to improve the safety and efficacy of temperate phages in a manner similar to that employed with strictly lytic phages. Furthermore, unique properties of temperate phages, including the capacity to integrate into the bacterial genome, can be exploited in singular therapeutic approaches. In the following paragraphs we debate both the limitations and the possible advantages of using temperate phages for therapy, and discuss the main strategies being pursued to do so.

\section{Limitations of Temperate Phages for Phage Therapy}

There are numerous concerns about the application of temperate phages for therapeutic purposes. Due to their intrinsic nature, temperate phages will often opt for a lysogenic life cycle (Box 1) in which they integrate into the bacterial genome and replicate in synchrony with the bacteria [6]. Therefore, the administration of temperate phages may not result in an immediate bactericidal effect. Also, when integrated in the bacterial chromosome, temperate phages may display superinfection immunity [15], making phage-sensitive bacteria insensitive to further phage infections.

Still, the major concern is the aptitude of temperate phages to mediate horizontal gene transfer between bacterial genomes via both generalized and specialized transduction [14]. In generalized transduction, fragments of bacterial DNA are packaged into the viral capsid during phage assembly inside the cell and are transferred to new hosts after subsequent phage infection [14]. It should be noted that this type of transduction can also be performed by lytic phages and is thus difficult to avoid in phage therapy. In specialized transduction, which occurs frequently with temperate phages, genes adjacent to the phage genome in the bacterial

\section{Box 1. Bacteriophage Lifestyles}

Bacteriophages are viruses that depend upon infection of a specific bacterial host for replication. Infection begins with the adsorption of the phage to specific bacterial receptors located at the cell surface; this causes ejection of the phage genome into the cell. The subsequent replication strategy depends on whether the phage is strictly lytic or temperate.

Strictly lytic phages follow a lytic life cycle in which, immediately upon genome ejection, the expression of phage early genes redirects the host metabolism to phage DNA replication and protein synthesis. Viral proteins are then assembled and the viral genome is packaged into the capsids. At the end of the lytic cycle, the production of phage late proteins, as holins and endolysins, leads to cell lysis for the release of progeny phages that can start another round of infection $[44,45]$.

Temperate phages follow a lysogenic life cycle in which they generally integrate their genome into the host chromosome where they remain quiescent, as prophages. The prophage is replicated along with the bacterial chromosome and transmitted by cell division to the daughter cells. This quiescent state can be maintained for long periods unless the cell is exposed to an environmental stress that can cause the prophage to be induced into a lytic life cycle [19,44].

Additionally, phages can assume a pseudolysogenic life cycle, in which the phage genome is carried in the host cells without propagation (Iytic cycle) or replication with the cell genome (lysogenic cycle). The phage's unintegrated genome is inherited by only one of the emerging progeny cells. This phenomenon is apparently caused by unfavourable growth conditions for the host cells, such as serious starvation, and is terminated when these conditions improve; the phage then restarts its development either through the lytic or the lysogenic life cycle $[46,47]$.

\section{Glossary}

Allele: one of two or more variants of a given gene that arises by mutation and is found in the same position on a chromosome.

Allelic exchange: replacement of a native allele with an alternative one carrying a mutation, by homologous recombination.

Bacteriophage: a virus that specifically infects and replicates within bacteria and archaea. Biofilm: microbial communities adhered to biotic or abiotic surfaces and embedded in a self-produced polymeric matrix.

CRISPR-Cas: a prokaryotic version of adaptive immunity, in which DNA fragments of invading DNA are captured into CRISPR repeats, which allow for cleavage of the invader DNA by a Cas nuclease upon a second invasion.

Dominant allele: an allele whose phenotype masks the contribution of another allele of the same gene at the same locus.

Endolysin: a phage-encoded enzyme, synthesized in the last stage of the phage lytic cycle, which cleaves the host cell wall for progeny release.

Endotoxin: a toxic molecule released from the cell wall of Gramnegative bacteria upon cell lysis. Fastidious: any organism with a complex nutritional requirement. Filamentous phage: a type of phage defined by its filament-like or rod-like shape, single-stranded DNA, and replication without host death.

Gibson assembly: a molecular cloning method that allows the assembly of multiple DNA fragments in a single-tube isothermal reaction. Horizontal gene transfer: the transfer of genetic material between organisms in the population other than by inheritance from ancestors (vertical transmission).

Immunogenicity: the ability of a substance to trigger an immune response.

Integrase: a viral enzyme that catalyses the integration of viral DNA into the host chromosome.

L-forms: cell-wall-deficient variants of normally walled bacteria which retain the ability to grow and divide. Lysogen: a bacterial cell that harbours a prophage integrated into 
chromosome can be erroneously excised together with the phage genome, and integrated into the chromosome of newly infected bacterial hosts [16-18].

Moreover, when temperate phages contain genes that the bacterial host can use to increase its virulence, a phenomenon of lysogenic conversion may occur (i.e., phage-mediated conversion of a nonvirulent strain into a virulent variant) $[14,19]$. Well known examples of this phenomenon are the dysentery-causing Escherichia coli O157:H7 that has acquired two prophages encoding the Shiga toxin [20], and the cholera pathogen Vibrio cholerae that has acquired a filamentous phage $(C T X \phi)$ encoding the cholera toxin [21]. Transduction also favours the dissemination of antibiotic-resistance genes [17] and other mobile genetic elements such as the pathogenicity islands in Staphylococcus aureus [22,23], thereby contributing to the evolution of bacterial pathogenicity.

\section{Advantages of Temperate Phages for Phage Therapy}

Despite the many concerns surrounding the therapeutic use of temperate phages, these phages also possess particularly interesting advantageous features. Temperate phages are very abundant in nature, with nearly half of the sequenced bacteria being lysogens [24], which makes them easy to find and isolate. Advances in next-generation sequencing technologies are particularly promising for this purpose, since the increasing number of genome sequences deposited in bioinformatics databases support the easier access, analysis, and identification of temperate phages by marker genes such as integrases [25]. The possibility of identifying temperate phages in bacterial genomes, instead of having to search for them in nature, is particularly useful for fastidious and anaerobic bacteria, where the isolation of strictly lytic phages has proven to be extremely difficult [26].

While the integration of temperate phages in bacterial genomes is generally regarded as a critical issue (as discussed earlier), it can also be explored for purposes in which it is desired. Recent advances in synthetic biology (Box 2) have made it possible, for example, to engineer temperate phages to deliver synthetic gene networks which interfere with important bacterial intracellular processes with the aim of causing bacterial cell death [27] or resensitizing the bacteria to antibiotics [28]. As this approach does not imply cell lysis, it reduces the risk of endotoxin release associated with the use of lytic phages.

It is also possible to tailor the genome of temperate phages to eliminate genes known to be involved in the maintenance of a lysogenic life cycle or in bacterial virulence [29,30]. By doing so, temperate phages become lytic and can be used as any other natural strictly lytic phage, or further improved by synthetic biology approaches. It should be noted, though, that most phage genes are of unknown function and may thus be involved in unrecognized undesirable events. This applies to both lytic and temperate phages, and highlights the need for further understanding of basic gene function to potentiate phage engineering approaches.

\section{How Temperate Phages Are Being Explored for Therapy}

While strictly lytic phages remain the preferred option for therapeutic purposes, a few studies have also begun to explore the potential of temperate phages (Table 1). The strategies employed are diverse, as summarized in Figure 1 (Key Figure) and detailed below.

\section{Strategies Using Natural (Nonengineered) Temperate Phages}

A few cases have been described in which temperate phages have been used for therapeutic purposes with interesting outcomes. Temperate phages MP22 and D3112, isolated from a clinical strain of Pseudomonas aeruginosa, were shown to reduce the swarming and twitching the chromosome or as a stable plasmid.

Lysogenic conversion (lysogenization): a change in the properties of a bacterial cell caused by the presence of a prophage. Microbiota: a community of microorganisms that typically inhabit a particular environment, for example, the human gut. Next-generation sequencing: a general term used to describe modern (non-Sanger-based) highthroughput DNA sequencing. Pathogenicity: the ability of an organism to cause disease/damage in a host.

Pathogenicity island: a region of the chromosome of a pathogenic bacterium coding for virulence genes normally absent from nonpathogenic strains of the same/closely related species, acquired by horizontal transmission.

Phage therapy: the use of phages to treat bacterial infections.

Selective pressure: external pressure that defines whether an organism will be more or less successful at surviving in a given environment.

Superinfection immunity: blocking of a second phage infection superimposed on the previous one. Synthetic biology: the design and construction of new biological systems, organisms, or devices for useful purposes.

Transduction: the process by which a phage transfers genetic material, other than its own, from one bacterium to another. Virulence: the severity or degree of damage caused by a pathogenic organism. 


\section{Box 2. Bacteriophage Engineering Tools}

Advances in the synthetic biology field have led to the development of new and more efficient genetic engineering tools that can be applied to build synthetic phages with new and/or improved functionalities. To date, several strategies have been successfully used to genetically engineer phage genomes.

- Homologous recombination. This technique enables recombination between heterologous DNA introduced in the host cell and the phage genomic DNA, when both sequences share regions of homology. Generally, a plasmid carrying regions of homology with the phage genome is introduced into the host cell and, upon phage infection, homologous recombination occurs between the plasmid and the phage genome leading to the generation of recombinant phages [48].

- Bacteriophage recombineering of electroporated DNA. This technique improves on homologous recombination by equipping the host cells with a plasmid encoding a recombinase protein that promotes high levels of recombination. The coelectroporation of phage DNA and dsDNA into the plasmid-carrying host cells results in high frequencies of engineered phages $[49,50]$.

- Yeast-based platform. This system uses Saccharomyces cerevisiae as an intermediate host for genetic manipulation. The phage genome or multiple overlapping PCR fragments spanning the entire phage genome are cotransformed with a linearized yeast artificial chromosome (YAC) into yeast cells. Since the YAC contains overhangs homologous to the ends of the phage genome, homologous recombination occurs between them, and the phage genome is captured in the YAC. The resultant YAC-phage DNA is then extracted from yeast cells and transformed into the host bacterial cells for the recovery of recombinant phages [7].

- Gibson assembly. Synthetic phages can be obtained by PCR amplification of phage genomes in multiple overlapping DNA fragments, which can then be assembled by Gibson and transformed into the bacterial host to release the mutant phages [30].

- CRISPR-Cas genome editing. This technique modifies the phage host cells with both a CRISPR-Cas9 plasmid and a donor plasmid containing the desired mutation with regions of homology to the phage DNA. Cells are infected with the phage, and the delivered genome is cleaved by the CRISPR-Cas9 nuclease at the desired site for the mutation, resulting in genome inactivation. Due to the presence of the donor DNA, recombination occurs between the Cas9cleaved ends of the phage DNA and the donor DNA. This restores the integrity of the genome and allows the recovery of the engineered phages [51].

motility of $P$. aeruginosa lysogens, respectively. Because the twitching motility is important for bacterial virulence, a significant reduction in bacterial loads and mortality was observed in models of peritonitis-sepsis and Drosophila melanogaster systemic infections when using phage D3112 [31]. An inhibition of bacterial swarming motility was also observed for $P$. aeruginosa phage DMS3, which also reduced biofilm formation by 83 of 86 lysogenized strains. Here, the effect was found to be dependent on a CRISPR region in the host, and may represent a novel mechanism of lysogenic conversion [32].

In an unrelated study, temperate phage ØCD27 infecting Clostridium difficile was capable of significantly reducing the $C$. difficile load in the colon and, surprisingly, preventing toxin production by this bacterium. However, lysogenization also increased spore production, which heightens the possibility of reoccurrence of $C$. difficile infection after phage treatment [33]. It is clear from these studies that, while positive outcomes can emerge from phage integration in the bacterial genome, the currently unpredictable nature of these effects prohibits the direct use of temperate phages in therapy.

The ability to lysogenize bacteria is given to temperate phages by a gene cluster that is responsible for integration (integrase) and maintenance (repressors of the lytic cycle) of the prophage. While temperate phages will usually retain this cluster, mutations may occur at low frequency which result in loss of the phage's capacity to lysogenize, thus creating lytic (or virulent, vir) mutants, which can be used as any other strictly lytic phage. A natural method for obtaining vir mutants has been patented [34]. Here, the temperate phage was treated with a mutagen (e.g., hydroxylamine) and grown together with its cultivating strain (i.e., the strain used for phage production) and its source strain (i.e., the strain from which the phage was isolated). Individual phage plaques obtained from this culture were tested against both strains. Assuming 
Table 1. Summary of Results from Studies Using Temperate Phages for Therapeutic Purposes

\begin{tabular}{|c|c|c|c|c|}
\hline Phage (host) & Application & Approach & Results & Refs \\
\hline \multicolumn{5}{|l|}{ Natural } \\
\hline $\begin{array}{l}\varnothing \mathrm{CD} 27 \\
\text { (Clostridium difficile) }\end{array}$ & Therapy & $\begin{array}{l}\text { Use of temperate phage for treatment of } \\
\text { C. difficile infections in the colon. }\end{array}$ & $\begin{array}{l}\text { Temperate phage } \varnothing \mathrm{CD} 27 \text { significantly reduced } \\
\text { bacterial load and prevented toxin production, but } \\
\text { increased spore production. }\end{array}$ & [33] \\
\hline $\begin{array}{l}\text { MP22 and D3112 } \\
\text { (Pseudomonas aeruginosa) }\end{array}$ & Therapy & $\begin{array}{l}\text { Isolation of temperate phages from } \\
\text { clinical } P \text {. aeruginosa isolates and } \\
\text { treatment of } P \text {. aeruginosa-induced } \\
\text { peritonitis-sepsis and Drosophila } \\
\text { melanogaster systemic infection. }\end{array}$ & $\begin{array}{l}\text { Temperate phages MP22 and D3112 reduced } \\
\text { swarming and twitching motility of } P \text {. aeruginosa } \\
\text { lysogens, respectively. Twitching motility is } \\
\text { important for bacterial virulence, so lysogenization } \\
\text { by D3112 significantly reduced mortality and } \\
\text { bacterial loads. }\end{array}$ & [31] \\
\hline \multicolumn{5}{|l|}{ Engineering } \\
\hline $\begin{array}{l}\phi E f 11 \\
\text { (Enterococcus faecalis) }\end{array}$ & $\begin{array}{l}\text { Improving lytic } \\
\text { activity }\end{array}$ & $\begin{array}{l}\text { Recombination of phage } \phi E f 11 \text { with a } \\
\text { defective prophage. }\end{array}$ & $\begin{array}{l}\text { Recombinant phage } \phi E f 11 \text { had higher lytic } \\
\text { activity and greater host range ( } 49 \% \text { of the strains) } \\
\text { than the wild-type phage ( } 6 \% \text { of the strains). }\end{array}$ & [29] \\
\hline $\begin{array}{l}\lambda \\
\text { (Escherichia coli) }\end{array}$ & $\begin{array}{l}\text { Restoring } \\
\text { antimicrobial } \\
\text { sensitivity }\end{array}$ & $\begin{array}{l}\text { Design of phage } \lambda \text { for the delivery of } \\
\text { dominant wild-type genes, responsible } \\
\text { for antibiotic sensitivity, to bacteria } \\
\text { possessing a mutated version of the gene } \\
\text { that conferred antibiotic resistance. }\end{array}$ & $\begin{array}{l}\text { Bacteria lysogenized by phage } \lambda \text { mutant had the } \\
\text { MIC values for streptomycin reduced from } \\
200 \mu \mathrm{g} / \mathrm{ml} \text { to } 1.56 \mu \mathrm{g} / \mathrm{ml} \text {; MIC values for nalidixic } \\
\text { acid decreased twofold. }\end{array}$ & [36] \\
\hline $\begin{array}{l}\text { B025 } \\
\text { (Listeria monocytogenes) }\end{array}$ & $\begin{array}{l}\text { Improving lytic } \\
\text { activity }\end{array}$ & \multirow{2}{*}{$\begin{array}{l}\text { Construction of synthetic phages using } \\
\text { Listeria L-forms to reboot the synthetic } \\
\text { genomes. }\end{array}$} & $\begin{array}{l}\text { Synthetic lytic variants of temperate phage B025 } \\
\text { had improved efficacy and induced less phage } \\
\text { resistance in bacterial cells. }\end{array}$ & [30] \\
\hline \multirow[t]{2}{*}{$\begin{array}{l}\text { PSA } \\
\text { (Listeria monocytogenes) }\end{array}$} & $\begin{array}{l}\text { Improving lytic } \\
\text { activity }\end{array}$ & & $\begin{array}{l}\text { A synthetic virulent mutant of temperate phage } \\
\text { PSA, armed with an endolysin gene, was able to } \\
\text { target phage-resistant bystander cells. }\end{array}$ & \\
\hline & $\begin{array}{l}\text { Restore antimicrobial } \\
\text { sensitivity }\end{array}$ & $\begin{array}{l}\text { Design of phage lambda for the delivery of } \\
\text { a CRISPR-Cas system targeting } \\
\beta \text {-lactamase genes. }\end{array}$ & $\begin{array}{l}\text { Bacteria lysogenized by phage } \lambda \text { mutant become } \\
\text { completely sensitive to streptomycin and } \\
\text { gentamicin. }\end{array}$ & [28] \\
\hline $\begin{array}{l}\text { ØSaBov } \\
\text { (Staphylococcus aureus) }\end{array}$ & $\begin{array}{l}\text { Specific gene } \\
\text { inactivation }\end{array}$ & $\begin{array}{l}\text { Design of phage } \varnothing \text { SaBov for the delivery } \\
\text { of a CRISPR-Cas } 9 \text { system targeting the } \\
\text { nuc gene that is present in all S. aureus } \\
\text { cells. }\end{array}$ & $\begin{array}{l}\text { In vitro, the phage mutant was able to kill all cells } \\
\text { after } 8 \mathrm{~h} \text { with no survivors emerging after } 24 \mathrm{~h} \text {. } \\
\text { In vivo, the phage mutant reduced the bacterial } \\
\text { load by more than two orders of magnitude. }\end{array}$ & [27] \\
\hline \multicolumn{5}{|l|}{ Cocktail formulation } \\
\hline $\begin{array}{l}\text { phiCDHM1 } \\
\text { phiCDHM2 } \\
\text { phiCDHM3 } \\
\text { phiCDHM4 } \\
\text { phiCDHM5 } \\
\text { phiCDHM6 } \\
\text { (Clostridium difficile) }\end{array}$ & Therapy & Combination of temperate phages. & $\begin{array}{l}\text { The combination of temperate phages was } \\
\text { capable of eradicating all bacterial cells after } 5 \mathrm{~h} \text { in } \\
\text { vitro. In vivo, bacterial colonization of the gut in a } \\
\text { hamster model of acute } C \text {. difficile infection was } \\
\text { reduced by four orders of magnitude and the } \\
\text { onset of symptoms was delayed by } 33 \mathrm{~h} \text {. }\end{array}$ & [41] \\
\hline $\begin{array}{l}\text { phiCDHM1 } \\
\text { phiCDHM2 } \\
\text { phiCDHM5 } \\
\text { phiCDHM6 } \\
\text { (Clostridium difficile) }\end{array}$ & $\begin{array}{l}\text { Improving lytic } \\
\text { activity }\end{array}$ & Combination of temperate phages. & $\begin{array}{l}\text { A cocktail of four temperate phages reduced the } \\
C \text {. difficile load in fermentation vessels, spiked } \\
\text { with combined faecal slurries from healthy } \\
\text { volunteers, by six orders of magnitude in a } \\
\text { prophylaxis regimen after } 5 \mathrm{~h} \text { and complete } \\
\text { eradication following prophylactic or remedial } \\
\text { regimens of } 24 \mathrm{~h} \text {. The cocktail concomitantly } \\
\text { increased the presence of commensal bacterial } \\
\text { species, which may provide protection from } \\
\text { further recolonization of } C \text {. difficile. }\end{array}$ & [42] \\
\hline $\begin{array}{l}\text { phi297 } \\
\text { (Pseudomonas aeruginosa) }\end{array}$ & $\begin{array}{l}\text { Improving lytic } \\
\text { activity }\end{array}$ & $\begin{array}{l}\text { Isolation of vir mutants to complement } \\
\text { phage cocktails. }\end{array}$ & $\begin{array}{l}\text { vir mutants were able to lyse mutant PAO1 cells } \\
\text { resistant to commercial phage cocktails. }\end{array}$ & [43] \\
\hline
\end{tabular}


Key Figure

Strategies for the Application of Temperate Phages in Therapy.

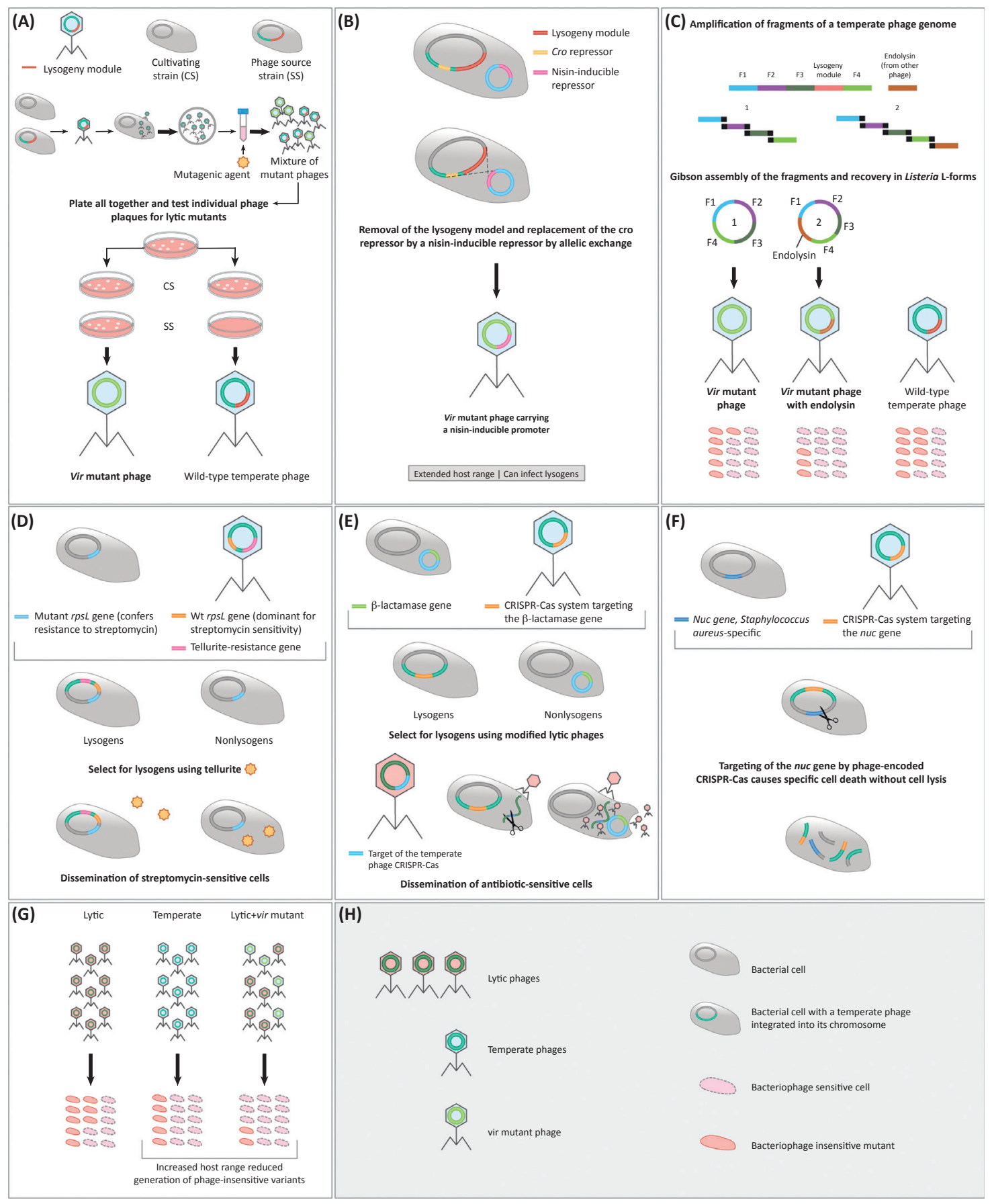

Trends in Microbiology 
that a vir mutant is one that can lytically infect a lysogenic host carrying the wild-type prophage, presumptive vir mutants can be identified by their ability to infect both the cultivating and source strain (Figure 1A).

\section{Strategies Using Engineered Temperate Phages}

Phage engineering has received increasing attention ever since synthetic biology evolved with new and more efficient techniques (Box 2). Whilst most engineering efforts have centred on strictly lytic phages, temperate phages have also been the subject of a few engineering experiments for phage therapy purposes. The most obvious approach consists of genetically modifying phages to become exclusively lytic. Zhang et al. observed that the Enterococcus faecalis temperate phage $\phi E f 11$ naturally recombined with a defective prophage in the host strain to yield a temperate phage with larger and somewhat clearer plaques than the original phage [29]. Using this variant with potentially enhanced lytic activity, the authors sought to create a vir mutant that could be used to treat serious $E$. faecalis infections without risk of lysogeny (Figure 1B). This was accomplished by deleting the genomic module responsible for the establishment of lysogeny, and by substituting the cro repressor with a nisin-inducible repressor. The modification was performed by allelic exchange between the phage variant incorporated in its host bacteria and a vector carrying the deletion construct and the nisin repressor. The resulting vir mutant was incapable of lysogeny and had a significantly extended host range (49\% of strains were infected by the mutant compared to $6 \%$ infected by the wildtype phage). The extended host range is most probably the result of the vir mutant showing productive infection (with the appearance of phage plaques) in strains previously lysogenized by the wt phage without signs of infection. Importantly, the substitution of the cro repressor with a nisin-inducible repressor rendered the vir phage insensitive to repression of the lytic cycle by the $c /$ repressor of the original $\phi E f 11$ when infecting lysogenized E. faecalis [29]. Clearly, the creation of vir mutants of otherwise temperate phages can easily extend the number and diversity of phages available for therapeutic purposes. But it appears that the genomic engineering required to create vir mutants can lead to phenotypic changes far beyond the inability to lysogenize the host bacteria, such as modification of the phage's host range. While an extension of the host range is an advantageous side effect, it is important to unmistakably identify all phenotypic changes to guarantee the safety of vir mutants in therapy. Using a distinct and fully synthetic approach, Kilcher et al. generated vir mutants of a temperate phage infecting Gram-positive bacteria (Figure 1C) [30]. The authors assembled, de novo, the genome of temperate Listeria phage B025, removing the lysogeny control functions that mediate genome integration and prophage maintenance [35]. The synthetic genome was assembled by Gibson assembly and subsequently rebooted in L-forms (i.e., bacterial variants that lack a cell wall) of Listeria monocytogenes. The L-form cells are vital for the successful reactivation of the synthetic phage DNA in Gram-positive cells by overcoming the cell wall barrier that obstructs

Figure 1. (A) vir variants of temperate phages can be selected using a mutagenic agent. (B) vir variants can be obtained by genetic engineering of temperate phages, also providing an opportunity for further modifications (e.g., replacement of the phage cro repressor by a nisin-inducible repressor to allow the vir variant to infect strains carrying the original temperate phage). (C) Genetic engineering of temperate phages of Gram-positive bacteria to make them strictly lytic or to improve particular features; this can be performed by amplification of the desired fragments of the phage genome and the genes to be inserted, which are Gibson assembled and rebooted in bacterial L-forms. (D) Temperate phages can be genetically modified to carry genes dominant for antibiotic sensitivity, and whose integration into the bacteria restores antibiotic susceptibility. By cointroducing a gene conferring resistance to tellurite, it is possible to select for antibiotic-sensitive lysogens using this toxic compound. (E) Temperate phages can be modified to carry CRISPR-Cas systems targeting antibiotic-resistance genes incorporated in bacterial plasmids. Combined with a lytic phage modified to be targeted by the same CRISPR-Cas system, it is possible to select for antibiotic-sensitive lysogens. (F) The CRISPR-Cas system integrated in temperate phages can be used to target genes specific for certain pathogenic bacterial species, causing cell death without lysis. (G) Phage cocktails of temperate phages, or of temperate/vir mutants and lytic phages, can result in increased host range and less generation of phage-insensitive mutants than cocktails of lytic phages. $(H)$ Symbol description. 
genome transfer into these cells [30]. Remarkably, as previously observed by Zhang et al. [29], the vir mutant phages displayed superior killing efficacy and were also less prone to generate phage-resistant bacterial variants than the wild-type phage. The reasons behind these phenotypic changes are still unclear, and certainly need to be addressed in upcoming studies. Additionally, Kilcher et al. applied the same strategy to L. monocytogenes temperate phage PSA with the additional incorporation of a gene encoding an endolysin into the phage genome for targeting of phage-resistant bystander cells. By doing so they demonstrated the potential of rational design of synthetic phages to improve their therapeutic properties [30].

Temperate phages can also be engineered to deliver synthetic gene networks. This approach exploits the natural capacity of the phage to integrate into the host bacterium's chromosome where it can express molecules of interest that interfere with intracellular processes. Using this concept, temperate phages have been modified to work as adjuvants to antibiotics. Inspired by a previous study with the filamentous phage M13 [12], Edgar et al. modified the temperate lambda phage to revert the pathogen to a streptomycin- and nalidixic acid-susceptible phenotype (Figure 1D) [36]. Resistance to these antibiotics has been associated with mutations in genes rps $L$ and gyrA. However, the wild-type genes are dominant sensitive alleles with regard to antibiotic resistance $[37,38]$, meaning that sensitivity will be restored if the wild-type genes are introduced in a resistant strain containing the mutant gene. Edgar and his coworkers explored this property by genetically engineering phage lambda to contain wild-type $r p s L$ and gyrA. Integration of the modified phage into a resistant strain conferred sensitivity to the two antibiotics in a dominant fashion. Additionally, a gene conferring resistance to tellurite was introduced into phage lambda so that sensitized bacteria could be enriched by selective pressure with this toxic compound. This strategy was proposed for dispersion of modified phages on hospital surfaces to gradually reverse the occurrence of drug-resistant pathogens [36]. However, this innovative approach is still limited to recessive antibiotic-resistance genes, and proper application would require the use of an alternative nontoxic form of selective pressure.

One such strategy was developed by Yosef et al. [28]. The authors combined modified temperate and lytic phages to reverse plasmid-associated antibiotic resistance in bacteria and select for antibiotic-sensitive variants (Figure 1E) [28]. Phage lambda was modified with the type I-E CRISPR-Cas system and a CRISPR array targeting conserved sequences of $\beta$-lactamase genes. The modified phage was capable of successfully reverting antibiotic resistance and preventing horizontal transfer of antibiotic resistance-encoding plasmids in consequence of plasmid degradation by the CRISPR-Cas system. Then, the lytic T7 phage was engineered to encode the sequences targeted by the CRISPR array introduced into phage lambda. By doing so, a selective advantage was given to the bacteria harbouring the prophage, that is, resistance to the lytic phage. When using both phages concomitantly, pathogens are sensitized by the temperate phage, and the sensitized population is selected and enriched by the lytic phage, which kills the antibiotic-resistant bacterial variants. As CRISPR spacers can be rationally designed to target any DNA sequence, this strategy could potentially be employed against any antibiotic resistance. As with the study of Edgar et al. [36], this strategy was proposed for use on hospital surfaces and hand sanitizers to facilitate replacement of antibiotic-resistance pathogens with sensitive variants [28]. Still, it is limited to antibiotic resistance encoded by plasmids and it cannot be applied to genome-encoded resistance.

More recently, Park et al. used temperate phages to deliver a CRISPR-Cas9 system designed to kill S. aureus cells (Figure 1F) [27]. The CRISPR-Cas9 system was developed to target the nuc gene uniquely present in all $S$. aureus cells, thus making the targeting specific. When tested in in vitro assays, the CRISPR-Cas9-carrying temperate phage ØSaBov demonstrated 
significantly enhanced killing efficacy specifically towards $S$. aureus, with rare occurrence of surviving mutants [27]. Similar enhanced efficacy of the modified phage was observed in a murine skin infection model, although here it was dependent on the water content of the skin. Since water activity is required for transcription and translation in S. aureus, so is the expression of the CRISPR-Cas9 system in these bacteria. Because of this limitation, the authors suggest the use of this strategy in topical applications supporting water activity, for example, infected tissues or contaminated medical surfaces [27].

Overall, it is clear that the engineering of temperate phages holds promise for therapeutic applications, either by tailoring their features towards lytic activity or by innovative exploration of their integrative properties.

\section{Cocktail Formulation}

Phage cocktails combine phages with distinct and often complementary features, for example, host range, to improve the efficacy of phage therapy and to reduce the emergence of phageresistant variants $[39,40]$. While most phage cocktail formulations have used strictly lytic phages, a few reports have emerged using temperate phages (Figure 1G). Due to a lack of strictly lytic phages targeting $C$. difficile, Nale et al. experimented with a cocktail combining different temperate phages [41]. While single-phage treatments caused the appearance of phage-resistant colonies, the use of the phage cocktail resulted in complete eradication of $C$. difficile in vitro and prevented the emergence of resistant and lysogenic variants [41]. Subsequent tests in a hamster model demonstrated the potential of the lytic-temperate phage cocktail by reducing bacteria colonization at $36 \mathrm{~h}$ postinfection and delaying the onset of symptoms by $33 \mathrm{~h}$ [41]. In another study, a cocktail of four temperate phages infecting $C$. difficile was shown to completely eradicate the bacterial load in fermentation vessels spiked with faecal slurries [42]. Curiously, treatment also resulted in the increased presence of commensal bacterial species, which may provide protection from further recolonization by C. difficile. Still, it is hard to predict whether similar effects would be obtained with other phages and bacterial species.

Additionally, Bourkal'tseva et al. demonstrated that the inclusion of a vir mutant of $P$. aeruginosa phage phi297 in a commercial mixture of strictly lytic phages significantly reduced the overall frequency of phage-resistant strains [43]. The reduction may result simply from the targeting of an additional host receptor by the vir mutant phage, which reduces the chances of bacteria developing resistance to all phages in the cocktail; but other unknown mechanisms may also be involved. This suggests that complementing cocktails of lytic phages with vir mutants of temperate phages may be a conceivable approach to prevent the rapid emergence of phage resistance.

\section{Concluding Remarks}

Strictly lytic phages will probably remain the main choice for phage therapy in the following years. Still, here we have highlighted that different approaches are being explored to make the therapeutic use of temperate phages viable and valuable. This is possible mainly because synthetic biology allows us to tailor phage genomes. vir mutants of temperate phages can now be easily generated, thereby increasing our arsenal of phages with strong lytic activity. But the phenotypic changes observed in vir mutants need to be further addressed since these changes were not restricted to loss of the mutant's capacity to integrate the bacterial genome.

Synthetic biology also creates the possibility of exploring the natural ability of temperate phages to integrate into bacterial genomes for therapeutic purposes. Only a few studies have begun to

\section{Outstanding Questions}

Temperate phages are still largely unexploited in therapy. Given their natural capacity to integrate into the bacterial genome, will temperate phages ever be used in their natural form, or will they need to be shaped by synthetic biology?

Considering the opportunities created by synthetic biology, which novel strategies will be developed for the use of temperate phages in therapy? In particular, how will integration of temperate phages be explored for further innovative strategies?

Is it possible to predict the effects of phage therapy with temperate phages in host/phage evolution by using, for example, evolutionary theories, genomic information, and mathematical modelling?

What mechanisms are behind the reduced frequency of phage-insensitive mutants generally reported for phage cocktails incorporating temperate phages or their lytic variants, compared to those containing strictly lytic phages only?

Is the combination of temperate phages with CRISPR-Cas systems a promising technology for controlling the spread of antibiotic resistance in hospital environments?

Given the prevalence of temperate phages in bacterial genomes, could these be a reliable option to replace natural lytic phages in future phage therapy approaches?

Would antibiotic resistance decrease with the implementation of phage therapy, using temperate and lytic phages, as a routine antibacterial treatment?

Would genetically manipulated phages be accepted by regulatory authorities as a promising antibacterial approach? 
explore this path, but have done so in very innovative and pertinent ways. The work so far can be separated into (i) studies using phage integration to cause direct cell death (without lysis) by interfering with essential metabolic paths, and (ii) studies that simply render bacteria less pathogenic or easier to control by using conventional therapeutic methods. Moreover, it is curious that several unrelated in vitro studies report strongly reduced frequency of emergence of phage-insensitive variants when incorporating temperate phages (natural or vir variants) in phage cocktails. Although the mechanism behind this phenomenon remains unknown, and the effect in vivo in combination with the immune system remains unclear, it seems increasingly relevant to explore combinations of temperate and strictly lytic phages in the future (see Outstanding Questions). Overall, temperate phages seem to be holding an immense therapeutic potential; we must now try to understand how to use it.

\section{Acknowledgments}

This work was supported by the Portuguese Foundation for Science and Technology (FCT) under the scope of the project PTDC/BBB-BSS/6471/2014 (POCl-01-0145-FEDER-016678), the strategic funding of UID/BIO/04469/2013 unit and COMPETE 2020 (POCl-01-0145-FEDER-006684). This work was also supported by BioTecNorte operation (NORTE-010145-FEDER-000004) funded by the European Regional Development Fund under the scope of Norte2020-Programa Operacional Regional do Norte. ARC and DPP were supported by FCT grants SFRH/BPD/94648/2013 and SFRH/BPD/ 116187/2016, respectively.

\section{Resources}

'https://hBTps://amr-review.org/sites/default/files/AMR\%20Review\%20Paper\%20-\%20Tackling\%20a\%20crisis\%20for \%20the\%20health\%20and\%20wealth\%20of\%20nations_1.pdf

\section{Supplemental Information}

Supplemental information associated with this article can be found online at doi:10.1016/j.tim.2018.10.008.

\section{References}

1. Zaman, S.B. et al. (2017) A review on antibiotic resistance: alarm bells are ringing. Cureus 9 , e1403

2. d'Herelle, F. (2007) On an invisible microbe antagonistic toward dysenteric bacilli: brief note by Mr F. d'Herelle, presented by $\mathrm{Mr}$ Roux. 1927. Res. Microbiol. 158, 553-554

3. Tan, S.Y. and Tatsumura, Y. (2015) Alexander Fleming (18811955): discoverer of penicillin. Singapore Med. J. 56, 366-367

4. Nilsson, A.S. (2014) Phage therapy-constraints and possibilities. Uppsala J. Med. Sci. 119, 192-198

5. Loc-Carrillo, C. and Abedon, S.T. (2011) Pros and cons of phage therapy. Bacteriophage 1, 111-114

6. Roach, D.R. et al. (2017) Synergy between the host immune system and bacteriophage is essential for successful phage therapy against an acute respiratory pathogen. Cell Host Microbe 22, 38-47.e34

7. Ando, H. et al. (2015) Engineering modular viral scaffolds for targeted bacterial population editing. Cell Syst. 1, 187-196

8. Hagens, S. et al. (2004) Therapy of experimental Pseudomonas infections with a nonreplicating genetically modified phage. Antimicrob. Agents Chemother. 48, 3817-3822

9. Matsuda, T. et al. (2005) Lysis-deficient bacteriophage therapy decreases endotoxin and inflammatory mediator release and improves survival in a murine peritonitis model. Surgery 137 , 639-646

10. Vitiello, C.L. et al. (2005) An amino acid substitution in a capsid protein enhances phage survival in mouse circulatory system more than a 1000 -fold. Virus Res. 114, 101-103

11. Lu, T.K. and Collins, J.J. (2007) Dispersing biofilms with engineered enzymatic bacteriophage. Proc. Natl. Acad. Sci. U. S. A. $104,11197-11202$

12. Lu, T.K. and Collins, J.J. (2009) Engineered bacteriophage targeting gene networks as adjuvants for antibiotic therapy. Proc. Natl. Acad. Sci. U. S. A. 106, 4629-4634

13. Oliveira, H. et al. (2015) Unexploited opportunities for phage therapy. Front. Pharmacol. 6, 180

14. Harrison, E. and Brockhurst, M.A. (2017) Ecological and evolutionary benefits of temperate phage: what does or doesn't kill you makes you stronger. Bioessays 39, 1700112

15. Dedrick, R.M. et al. (2017) Prophage-mediated defense against viral attack and viral counter-defense. Nat. Microbiol. 2, 16251 16251

16. Penadés, J.R. et al. (2015) Bacteriophage-mediated spread of bacterial virulence genes. Curr. Opin. Microbiol. 23, 171-178

17. Haaber, J. et al. (2016) Bacterial viruses enable their host to acquire antibiotic resistance genes from neighbouring cells. Nat. Commun. 7, 13333

18. Colavecchio, A. et al. (2017) Bacteriophages contribute to the spread of antibiotic resistance genes among foodborne pathogens of the Enterobacteriaceae family - a review. Front. Microbiol. 8, 1108

19. Davies, E.V. et al. (2016) The role of temperate bacteriophages in bacterial infection. FEMS Microbiol. Lett. 363, fnw0155

20. Shaikh, N. and Tarr, P.I. (2003) Escherichia coli O157:H7 shiga toxin-encoding bacteriophages: integrations, excisions, truncations, and evolutionary implications. J. Bacteriol. 185, 3596-3605

21. Waldor, M.K. and Mekalanos, J.J. (1996) Lysogenic conversion by a filamentous phage encoding cholera toxin. Science 272 , 1910-1914

22. Chen, J. and Novick, R.P. (2009) Phage-mediated intergeneric transfer of toxin genes. Science 323, 139-141 
23. Dearborn, A.D. and Dokland, T. (2012) Mobilization of pathogenicity islands by Staphylococcus aureus strain Newman bacteriophages. Bacteriophage 2, 70-78

24. Touchon, M. et al. (2016) Genetic and life-history traits associated with the distribution of prophages in bacteria. ISME J. 10, 2744 2754

25. Howard-Varona, C. et al. (2017) Lysogeny in nature: mechanisms, impact and ecology of temperate phages. ISME J. 11, 1511

26. Hargreaves, K.R. and Clokie, M.R.J. (2014) Clostridium difficile phages: still difficult? Front. Microbiol. 5, 184

27. Park, J.Y. et al. (2017) Genetic engineering of a temperate phagebased delivery system for CRISPR/Cas9 antimicrobials against Staphylococcus aureus. Sci. Rep. 7, 44929

28. Yosef, I. et al. (2015) Temperate and lytic bacteriophages programmed to sensitize and kill antibiotic-resistant bacteria. Proc. Natl. Acad. Sci. U. S. A. 112, 7267-7272

29. Zhang, H. et al. (2013) Genetic modifications to temperate Enterococcus faecalis phage $\phi \mathrm{Ef1} 1$ that abolish the establishment of lysogeny and sensitivity to repressor, and increase host range and productivity of lytic infection. Microbiology 159, 10231035

30. Kilcher, S. et al. (2018) Cross-genus rebooting of custom-made, synthetic bacteriophage genomes in L-form bacteria. Proc. Natl. Acad. Sci. U. S. A. 115, 567-572

31. Chung, I.-Y. et al. (2012) Antibacterial efficacy of temperate phage-mediated inhibition of bacterial group motilities. Antimicrob. Agents Chemother. 56, 5612-5617

32. Zegans, M.E. et al. (2009) Interaction between bacteriophage DMS3 and host CRISPR region inhibits group behaviors of $P$ Seudomonas aeruginosa. J. Bacteriol. 191, 210-219

33. Meader, E. et al. (2013) Evaluation of bacteriophage therapy to control Clostridium difficile and toxin production in an in vitro human colon model system. Anaerobe 22, 25-30

34. Rapson, M.E. et al. (2003) Bacteriophages useful for therapy and prophylaxis of bacterial infections, WO/2003/080823

35. Dorscht, J. et al. (2009) Comparative genome analysis of Listeria bacteriophages reveals extensive mosaicism, programmed translational frameshifting, and a novel prophage insertion site. J. Bacteriol. 191, 7206-7215

36. Edgar, R. et al. (2012) Reversing bacterial resistance to antibiotics by phage-mediated delivery of dominant sensitive genes. Appl. Environ. Microbiol. 78, 744-751
37. Lederberg, J. (1951) Streptomycin resistance: a genetically recessive mutation. J. Bacteriol. 61, 549-550

38. Robillard, N.J. (1990) Broad-host-range gyrase A gene probe. Antimicrob. Agents Chemother. 34, 1889-1894

39. Chan, B.K. et al. (2013) Phage cocktails and the future of phage therapy. Future Microbiol. 8, 769-783

40. Yen, M. et al. (2017) A cocktail of three virulent bacteriophages prevents Vibrio cholerae infection in animal models. Nat. Commun. 8, 14187

41. Nale, J.Y. et al. (2016) Bacteriophage combinations significantly reduce Clostridium difficile growth in vitro and proliferation in vivo. Antimicrob. Agents Chemother. 60, 968-981

42. Nale, J. et al. (2018) Efficacy of an optimised bacteriophage cocktail to clear Clostridium difficile in a batch fermentation model. Antibiotics 7, 13

43. Bourkal'tseva, M.V. et al. (2011) Bacteriophage phi297, a new species of Pseudomonas aeruginosa temperate phages with a mosaic genome: potential use in phage therapy. Russ. J. Genet. 47, 794-798

44. Guttman, B. et al. (2004) Basic phage biology. In Bacteriophages: Biology and Applications (Kutter, E. and Sulakvelidze, A., eds), CRC Press

45. Drulis-Kawa, Z. et al. (2012) Learning from bacteriophages advantages and limitations of phage and phage-encoded protein applications. Curr. Protein Pept. Sci. 13, 699-722

46. Los, M. et al. (2003) A role for bacteriophage T4 rl gene function in the control of phage development during pseudolysogeny and in slowly growing host cells. Res. Microbiol. 154, 547-552

47. Łoś, M. and Wegrzyn, G. (2012) Chapter 9 - Pseudolysogeny. In Advances in Virus Research (Małgorzata, Ł. and Wacław, T.S. eds), pp. 339-349, Academic Press

48. Le, S. et al. (2013) Mapping the tail fiber as the receptor binding protein responsible for differential host specificity of Pseudomonas aeruginosa bacteriophages PaP1 and JG004. PLoS One 8 e68562

49. Shin, H. et al. (2014) Genomic investigation of lysogen formation and host lysis systems of the Salmonella temperate bacteriophage SPN9CC. Appl. Environ. Microbiol. 80, 374-384

50. Marinelli, L.J. et al. (2012) Recombineering: a powerful tool for modification of bacteriophage genomes. Bacteriophage 2, 5-14

51. Tao, P. et al. (2017) Engineering of bacteriophage T4 genome using CRISPR-Cas9. ACS Synth. Biol. 6, 1952-1961 